\title{
Robust Mean Traveltime Curves in 2D Transmission Tomographic Surveys
}

\author{
Juan L. Fernández Martínez • \\ Luis M. Pedruelo González • James W. Rector
}

Received: 22 October 2009 / Accepted: 4 February 2010 / Published online: 12 March 2010

(C) The Author(s) 2010. This article is published with open access at Springerlink.com

\begin{abstract}
Acoustic tomography methods belong to the class of nondestructive inspection techniques and are used in engineering applications. One of the main issues for these methods is the direct arrival, which can be noisy or can be affected by scattering or other propagation effects. In this paper, we present the mathematical deduction and analysis of the so-called robust mean traveltime curves-the median, $p$-percentiles, inter-quartile range and minimum absolute deviation-for homogeneous isotropic or elliptical anisotropic media. Robust mean traveltime curves are a simple model used to describe the variation of the traveltime statistical descriptors for the different gathering subsets as a function of a gather index, and generalize the mean traveltime curves introduced in the past to the case of robust statistics. These curves admit analytical expression for zonal isotropic and elliptical anisotropic media explored via rectangular or irregular acquisition geometries, and thus, apply to 2D acoustic transmission tomography experiments conducted in relatively homogeneous blocks. The robust mean traveltime curves are more resistant to the presence of outliers, and thus they are preferred to infer background velocity models which can be taken into account in the resolution of the tomographic inverse problem. The property of robust descriptors to find blocky solutions in presence of velocity heterogeneities is also illustrated. Finally, we show the application of this methodology to a granitic medium (Febex Project, Nagra, Switzerland).
\end{abstract}

J.L. Fernández Martínez (凶) · L.M. Pedruelo González

Departamento de Matemáticas, Universidad de Oviedo, C/Calvo Sotelo, 33007 Oviedo, Spain e-mail: jlfm@uniovi.es

J.L. Fernández Martínez · J.W. Rector

Department of Civil and Environmental Eng., UC-Berkeley, Berkeley, CA 94720-1710, USA

J.L. Fernández Martínez

Department of Energy Resources Eng., Stanford University, Stanford, CA 94305-2220, USA 
Keywords Inverse problems · Transmission tomography · Mean traveltime curves · Robust fitting

\section{Introduction}

Tomographic inversion techniques have been widely used in very different geological engineering applications, and consist of iteratively finding the velocity field of a geological medium that better fits the traveltime data acquired from their boundaries. Different numerical methods have been reported in the literature to solve the transmission tomographic inverse problem, including biomedical reconstruction techniques (Gordon 1974; Dines and Lytle 1979; Ivansson 1986), conjugate gradient methods (Scales 1987), and least-squares algorithms (Berryman 1991). Since the tomographic inverse problem is ill-posed, it is crucial to have methodologies to analyze the traveltime quality on hand in order to identify and filter possible errors, and to infer prior velocity information to win stability at inversion. The Mean Traveltime Curves (MTC) is a simple methodology that has been designed to accomplish this task by analyzing the data variability the source and receiver gathers at the preinversion stage. These curves describe the variation of the main statistical descriptors (mean and standard deviation) of the experimental traveltimes for the different gathering subsets as a function of their gather index (Fernández-Martínez et al. 2006). The theoretical mean traveltime curves assume that the geological medium can be divided into homogeneous layers, either isotropic or elliptical anisotropic (Fernández-Martínez and Pedruelo-González 2008). Least-squares fitting of the empirical traveltime curves using the theoretical models allows the inference of background velocity models valid at experiment scale, which can be taken incorporated in the resolution of the inverse problem as regularization term. In the case of elliptical anisotropic media, the mean traveltime curves are a simple method to infer the anisotropic parameters from traveltime data at preinversion stage (Fernández-Martínez et al. 2009a).

These curves can also be useful in the detection of outliers. Since our first paper devoted to this subject, we have shown that different kind of data errors have different signatures that can be used to discriminate them from geological heterogeneities (Fernández-Martínez et al. 2006). Also, sensibility analysis performed on these curves showed two important conclusions: (i) the mean curve is less prone to errors than the standard deviation curve, and thus is more suitable for velocity identification (Fernández-Martínez et al. 2006; Fernández-Martínez and PedrueloGonzález 2008); and (ii) data errors can be misinterpreted as high anomalous values of anisotropy (Fernández-Martínez and Pedruelo-González 2008). A common alternative in inversion to diminish the effect of the data errors is to make use of the L1 norm (Scales and Gersztenkorn 1988). The use of this norm is implicitly related to long-tail probability distribution (exponential) for the misfit error. These kinds of distributions are able to anticipate outliers without drastically modifying its center of mass. This idea has led us to propose the robust mean traveltime curves that describe the $p$-percentiles and the associated dispersion descriptors (inter-quartile range and minimum absolute deviation) of the experimental traveltime distribution. 
In this paper, we present the theoretical deduction and analysis of the robust mean traveltime curves for the case of a layered isotropic or elliptical anisotropic media explored via any arbitrary recording geometry. As mentioned above, these statistical measures belong to the category of robust statistics, since they are less sensible to the presence of outliers than the classical descriptors (mean and standard deviation). These curves have already been implemented in AMTCLAB (Fernández-Martínez et al. 2009a). We show how the anisotropy of the geological medium and the irregularity of the recording geometry affect the shape of these curves. Sensitivity analysis to data errors and different geological heterogeneities is also performed by means of synthetic cases. As the main conclusion we find that the mean, median, and upperquartile curves are less prone to errors than the mean curve. Similar conclusions are also reached by the application of this methodology to the Grimsel data set. This methodology has also been recently applied to analyze the anisotropy of a rock massif in France studied by GPR (Ground Penetrating Radar) transmission data (FernándezMartínez et al. 2009b).

\section{The Robust Mean Traveltime Curves in the Isotropic Case}

Let $\Omega$ be homogeneous and isotropic medium with constant velocity to be studied by transmission tomography. Let us also assume that we have two lines of sources and receivers located on the boundary of $\Omega$. Gathering subsets can be defined grouping together traveltimes arriving to each receiver (common receiver gathering) and those emitted from each source. Mean traveltime curves describe analytically the variation of the main statistical parameters (mean and standard deviation) of the different gathering subsets as a function of the gather distance (Fernández-Martínez et al. 2006). Robust mean traveltime curves refer to the variation of the different $p$-percentiles and related measures of dispersion (inter-quartile range and minimum absolute deviation) for the above mentioned gathering subsets. It will be shown that these measures are more robust in presence of data errors.

\subsection{The Rectangular Recording Geometry Case}

Let $\Omega$ be a homogeneous isotropic domain explored by means of a rectangular recording geometry (sources and receivers placed along two parallel boreholes) as shown in Fig. 1(a). Seismic rays follow straight lines. Due to the isotropy and homogeneity, the traveltime distributions in each source gather, $x_{s}$, follow the hyperbolic law

$$
t\left(\xi ; x_{s}\right)=\sqrt{t_{0}^{2}+\frac{\left(\xi-x_{s}\right)^{2}}{V^{2}}},
$$

where $\xi$ describes the receiver positions with respect to the gather origin, $V$ is the constant velocity of the medium, and $t_{0}$ is the minimum traveltime in the gathering, which remains the same for all gathers due to the rectangular acquisition geometry. 


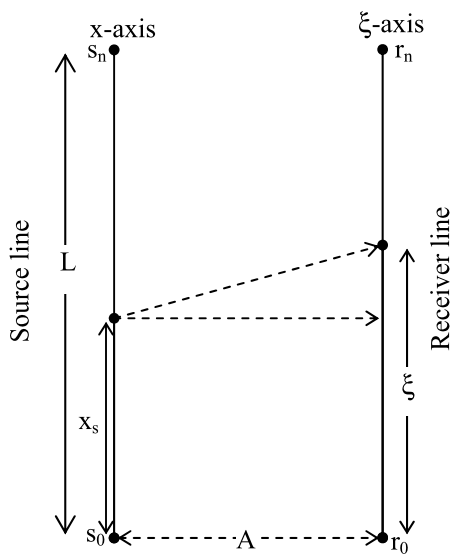

(a)

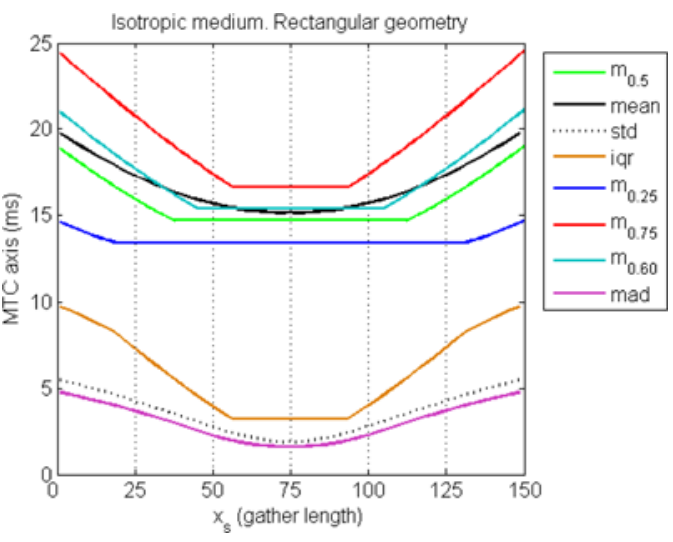

(b)

Fig. 1 (a) Rectangular recording geometry and variables involved in the mean traveltime curve analysis. (b) Robust MTC curves in relation to the mean and standard deviation curves

The following statistical descriptors of the traveltime distribution for different gathering subsets are of interest:

\section{1 .1 p-Percentiles}

The $p$-percentile, $m_{p}$, of the traveltime distribution,

$$
t\left(\xi ; x_{s}\right)=\sqrt{t_{0}^{2}+\frac{\left(\xi-x_{s}\right)^{2}}{V^{2}}},
$$

is implicitly defined as

$$
P\left(t\left(\xi ; x_{s}\right)<m_{p}\right)=p \in[0,1]
$$

where $p$ is the probability threshold corresponding to $m_{p}$. For instance, the median corresponds to $p=0.5$, and the lower and upper percentiles to $p=0.25$ and $p=0.75$.

Considering that the spatial variable $\xi$ is distributed uniformly on the borehole length, $L(\xi \rightarrow U(0, L))$, this probability can be calculated as follows

$$
P\left(t\left(\xi ; x_{s}\right)<m_{p}\right)=P\left(x_{1}<\xi<x_{2}\right)=\int_{x_{1}}^{x_{2}} \frac{1}{L} d x,
$$

where

$$
\begin{aligned}
& x_{1}=\max \left\{0, x_{s}-\sqrt{\left(m_{p}^{2}-t_{0}^{2}\right)} \cdot V\right\}, \\
& x_{2}=\min \left\{L, x_{s}+\sqrt{\left(m_{p}^{2}-t_{0}^{2}\right)} \cdot V\right\} .
\end{aligned}
$$


In this calculation, three cases arise

Case 1.

$$
0<x_{s}<\frac{p L}{2} \Longrightarrow m_{p}\left(x_{s}\right)=\sqrt{t_{0}^{2}+\frac{\left(p L-x_{s}\right)^{2}}{V^{2}}},
$$

Case 2.

$$
\frac{p L}{2}<x_{s}<L-\frac{p L}{2} \Longrightarrow m_{p}\left(x_{s}\right)=\sqrt{t_{0}^{2}+\frac{(p L)^{2}}{4 V^{2}}},
$$

Case 3.

$$
L-\frac{p L}{2}<x_{s}<L \Longrightarrow m_{p}\left(x_{s}\right)=\sqrt{t_{0}^{2}+\frac{\left((p-1) L+x_{s}\right)^{2}}{V^{2}}} .
$$

As a main conclusion, the $p$-percentile curves are symmetric with respect to the middle of the gather line, $x_{s}=\frac{L}{2}$, and have a sill interval whose length is $L(1-p)$ of constant value, $m_{p c}$, which is related to the isotropic velocity

$$
V=\frac{\sqrt{A^{2}+\left(\frac{p L}{2}\right)^{2}}}{m_{p c}} .
$$

This formula provides a simple and robust method (resistant to outliers) to determine the isotropic velocity, $V$, since it uses the $p$-percentile sill value, $m_{p c}$, which is the traveltime at position $x_{p}=x_{s}+\frac{p L}{2}$, and is the same for all the gathers on the interval $\left[\frac{p L}{2}, L-\frac{p L}{2}\right]$.

The median curve, $m_{p=1 / 2}\left(x_{s}\right)$, is a particular case and provides the trajectory of the center of the traveltime distribution as a function of the gather index, $x_{s}$. This curve is a symmetric curve, has a sill whose length is $\frac{L}{2}$, and constant value, $m_{1 / 2}$, which is related to the isotropic velocity as follows

$$
V=\frac{\sqrt{A^{2}+\left(\frac{L}{4}\right)^{2}}}{m_{1 / 2}} .
$$

\subsubsection{The Inter-Quartile Range}

The inter-quartile range is the difference

$$
\operatorname{iqr}\left(x_{s}\right)=m_{3 / 4}\left(x_{s}\right)-m_{1 / 4}\left(x_{s}\right) \text {. }
$$

Taking into account the analytical expression of the $p$-percentiles, it is easy to show that the inter-quartile range for gather subset, $x_{s}$, has the following expression

Case 1.

$$
0<x_{s}<\frac{L}{8} \Longrightarrow \operatorname{iqr}\left(x_{s}\right)=\sqrt{t_{0}^{2}+\frac{\left(\frac{3 L}{4}-x_{s}\right)^{2}}{V^{2}}}-\sqrt{t_{0}^{2}+\frac{\left(\frac{L}{4}-x_{s}\right)^{2}}{V^{2}}},
$$


Case 2.

$$
\frac{L}{8}<x_{s}<\frac{3 L}{8} \Longrightarrow \operatorname{iqr}\left(x_{s}\right)=\sqrt{t_{0}^{2}+\frac{\left(\frac{3 L}{4}-x_{s}\right)^{2}}{V^{2}}}-\sqrt{t_{0}^{2}+\frac{\left(\frac{L}{8}\right)^{2}}{V^{2}}},
$$

Case 3.

$$
\frac{3 L}{8}<x_{s}<\frac{5 L}{8} \Longrightarrow \operatorname{iqr}\left(x_{s}\right)=\sqrt{t_{0}^{2}+\frac{\left(\frac{3 L}{8}\right)^{2}}{V^{2}}}-\sqrt{t_{0}^{2}+\frac{\left(\frac{L}{8}\right)^{2}}{V^{2}}}
$$

Case 4.

$$
\frac{5 L}{8}<x_{s}<\frac{7 L}{8} \Longrightarrow \operatorname{iqr}\left(x_{s}\right)=\sqrt{t_{0}^{2}+\frac{\left(x_{s}-\frac{L}{4}\right)^{2}}{V^{2}}}-\sqrt{t_{0}^{2}+\frac{\left(\frac{L}{8}\right)^{2}}{V^{2}}},
$$

Case 5.

$$
\frac{7 L}{8}<x_{s}<L \Longrightarrow \operatorname{iqr}\left(x_{s}\right)=\sqrt{t_{0}^{2}+\frac{\left(x_{s}-\frac{L}{4}\right)^{2}}{V^{2}}}-\sqrt{t_{0}^{2}+\frac{\left(x_{s}-\frac{3 L}{4}\right)^{2}}{V^{2}}} .
$$

The iqr curve has a sill whose length is $\frac{L}{4}$ and constant value, iqr $_{c}$, related to the isotropic velocity as follows

$$
V=\frac{\sqrt{A^{2}+\left(\frac{3 L}{8}\right)^{2}}-\sqrt{A^{2}+\left(\frac{L}{8}\right)^{2}}}{\operatorname{iqr}_{c}} .
$$

This formula can be generalized to any other percentile range, $\left[m_{p 1}, m_{p 2}\right]$, by

$$
\operatorname{iqp}\left(x_{s}\right)=m_{p_{2}}\left(x_{s}\right)-m_{p_{1}}\left(x_{s}\right) \text {. }
$$

The sill length is in this case $L\left(1-p_{2}\right)$, and the corresponding constant value, iqp , is related to the isotropic velocity in the following way

$$
V=\frac{\sqrt{A^{2}+\left(\frac{p_{2} L}{2}\right)^{2}}-\sqrt{A^{2}+\left(\frac{p_{1} L}{2}\right)^{2}}}{\operatorname{iqp}_{c}} .
$$

\subsubsection{The Minimum Absolute Deviation (mad)}

The minimum absolute deviation is

$$
\operatorname{mad}\left(x_{s}\right)=\frac{1}{L} \int_{0}^{L}\left|t\left(\xi ; x_{s}\right)-m_{1 / 2}\left(x_{s}\right)\right| d \xi,
$$

i.e., the mean absolute distance of $t\left(\xi ; x_{S}\right)$ to the median, $m_{1 / 2}\left(x_{S}\right)$, for each source gather, $x_{s}$. 
The minimum absolute deviation has the following expression

Case 1.

$$
\begin{aligned}
& 0<x_{s}<\frac{p L}{2}, \\
& \quad \operatorname{mad}\left(x_{s}\right)=\frac{1}{L} \int_{0}^{L p}\left(m_{p}\left(x_{s}\right)-t\left(\xi ; x_{s}\right)\right) d \xi+\frac{1}{L} \int_{L p}^{L}\left(t\left(\xi ; x_{s}\right)-m_{p}\left(x_{s}\right)\right) d \xi,
\end{aligned}
$$

Case 2.

$$
\begin{aligned}
\frac{p L}{2}<x_{s}<L & -\frac{p L}{2} \\
\operatorname{mad}\left(x_{s}\right)= & \frac{1}{L} \int_{0}^{x_{s}-\frac{L p}{2}}\left(t\left(\xi ; x_{s}\right)-m_{p}\left(x_{s}\right)\right) d \xi \\
& +\frac{1}{L} \int_{x_{s}-\frac{L p}{2}}^{x_{s}+\frac{L p}{2}}\left(m_{p}\left(x_{s}\right)-t\left(\xi ; x_{s}\right)\right) d \xi \\
& +\frac{1}{L} \int_{x_{s}+\frac{L p}{2}}^{L}\left(t\left(\xi ; x_{s}\right)-m_{p}\left(x_{s}\right)\right) d \xi
\end{aligned}
$$

Case 3.

$$
\begin{aligned}
L-\frac{p L}{2}<x_{s} & <L, \\
\operatorname{mad}\left(x_{s}\right)= & \frac{1}{L} \int_{0}^{L(1-p)}\left(t\left(\xi ; x_{s}\right)-m_{p}\left(x_{s}\right)\right) d \xi \\
& +\frac{1}{L} \int_{L(1-p)}^{L}\left(m_{p}\left(x_{s}\right)-t\left(\xi ; x_{s}\right)\right) d \xi
\end{aligned}
$$

These integrals can be analytically calculated and can be put in relation with the percentile, $m_{p}$, and the mean traveltime values, $\bar{t}_{[a, b]}$, calculated over certain gather intervals $[a, b]$ where the percentile function is defined

Case 1.

$$
\operatorname{mad}\left(x_{s}\right)=m_{p}(2 p-1)+\bar{t}_{[0, L]}-2 p \bar{t}_{[0, L p]},
$$

Case 2.

$$
\operatorname{mad}\left(x_{s}\right)=m_{p}(2 p-1)+\bar{t}_{[0, L]}-2 p \bar{t}_{\left[x_{s}-\frac{L p}{2}, x_{s}+\frac{L p}{2}\right]},
$$

Case 3.

$$
\operatorname{mad}\left(x_{p}\right)=m_{p}(2 p-1)+\bar{t}_{[0, L]}-2 p \bar{t}_{[L(1-p), L]} .
$$

The mad curve does not reach a sill, it is a decreasing-increasing curve with its minimum in the middle of the gather line (in the isotropic case). Also, this curve has 


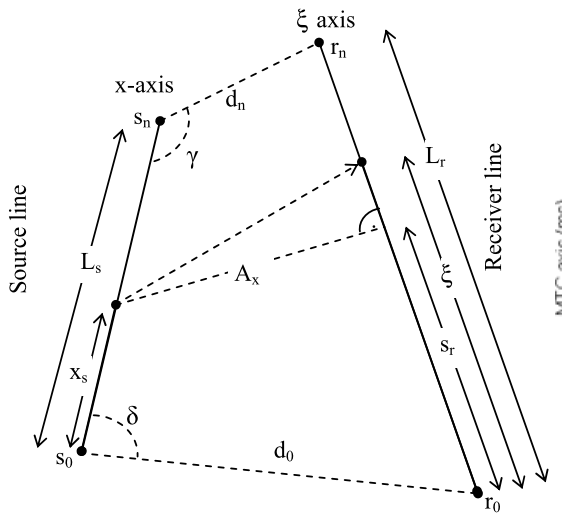

(a)

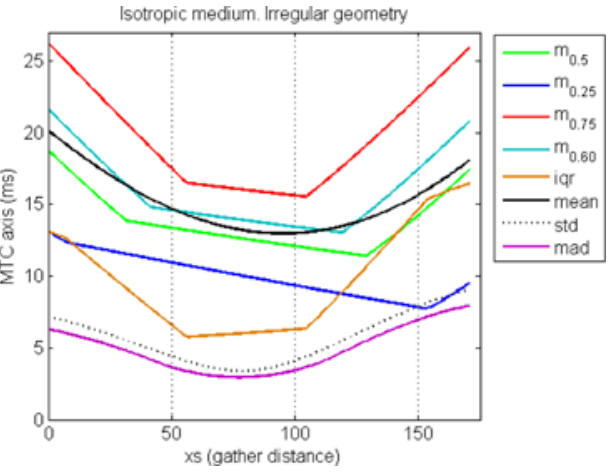

(b)

Fig. 2 (a) Parameters in the irregular recording geometry case. (b) Robust MTC curves compared to the mean and standard deviation curves. The medium is in this case isotropic with $V=5.4 \mathrm{~km} / \mathrm{s}$. The recording geometry parameters are: $d_{0}=66 \mathrm{~m}, d_{n}=32 \mathrm{~m}, L_{r}=191 \mathrm{~m}$, and $L_{s}=170 \mathrm{~m}$

two inflexion points located at $\frac{p L}{2}$ and $L-\frac{p L}{2}$, respectively, i.e., the $x_{s}$-values where the mad function changes.

Figure 1(b) shows the median, the lower and upper quartile, the inter-quartile range, and the minimum absolute deviation for an isotropic and homogeneous medium explored with a rectangular recording configuration $(L=140 \mathrm{~m}, V=$ $5.4 \mathrm{~km} \mathrm{~s}^{-1}$ and $A=70 \mathrm{~m}$ ), compared to the mean and standard deviation curves. The mean curve is close to the 0.6 percentile curve in this case, and the standard deviation to the mad curve.

\subsection{The Irregular Recording Geometry Case}

The above mentioned relationships can be generalized to an isotropic medium explored with an irregular recording geometry. Analytical expressions are identical to those in the rectangular case with the following identifications: $x_{s} \rightarrow s_{r}, L \rightarrow L_{r}$, $A \rightarrow A_{x}$. Calculations depend on recording geometry parameters $d_{0}, d_{n}, L_{r}, L_{s}$, where $d_{0}$ is the distance between the source in $x=x_{0}$ and the receiver in $\xi=\xi_{0}$, $d_{n}$ is the distance between the source in $x=x_{n}$ and the receiver in $\xi=\xi_{n}, A_{x}$ is the distance between the source in $x=x_{s}$ and the receiver borehole, $L_{r}$ is the receiver borehole length, and $L_{S}$ is the source borehole length. Development and deduction of the robust mean traveltime curves for irregular recording geometries is provided in Appendix A. Figure 2 shows all the variables involved in an irregular recording geometry and the shape of the robust traveltime curves we get for this case. As it can be observed, the irregular configuration provokes asymmetry of these curves. The sill also becomes a dipping straight line, since the $p$-percentile depends on the parameter $A_{x}$ for the gathering position, $x_{s}$. 


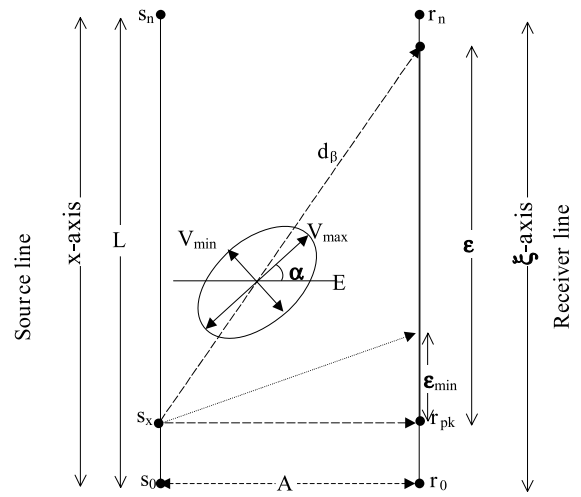

(a)

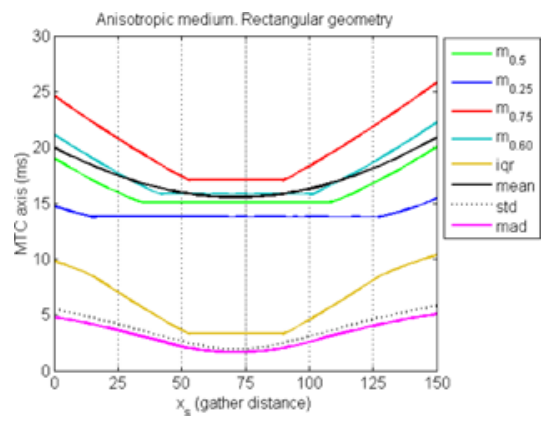

(b)

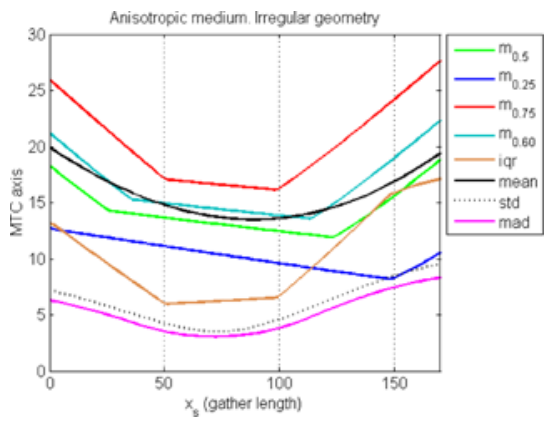

(c)

Fig. 3 (a) Parameters in the anisotropic case for a rectangular recording geometry. (b) Robust mean traveltime curves for an anisotropic medium with $V_{\max }=5.4 \mathrm{~km} / \mathrm{s}, \lambda=0.95$, and $\alpha=45\left(E 45^{\circ} N\right)$. (c) Robust mean traveltime curves for the same anisotropic medium and an irregular configuration geometry (same as in Fig. 2)

\section{The Robust Mean Traveltime Curves in the Anisotropic Case}

Fernández-Martínez and Pedruelo-González (2008) generalized the mean traveltime curves for media with an elliptical velocity model explored either with a rectangular or irregular recording geometry (Fig. 3). In this section, we present the robust mean traveltime curves for these two cases.

\subsection{The Rectangular Recording Geometry Case}

In this case, the traveltime variations in each source gather, $x_{s}$, can be described through the following relationship (Fernández-Martínez and Pedruelo-González 2008) 
$t\left(\xi ; x_{s}\right)=\sqrt{\frac{\left(1+\lambda^{2} \tan ^{2} \alpha\right)\left(\xi-x_{s}\right)^{2}+2 A \tan \alpha\left(\lambda^{2}-1\right)\left(\xi-x_{s}\right)+A^{2}\left(\lambda^{2}+\tan ^{2} \alpha\right)}{\lambda^{2}\left(1+\tan ^{2} \alpha\right) V_{\max }^{2}}}$.

By introducing the variables

$$
\begin{aligned}
& a=\frac{\left(1+\lambda^{2} \tan ^{2} \alpha\right)}{\lambda^{2}\left(1+\tan ^{2} \alpha\right) V_{\max }^{2}}, \quad b=\frac{2 A \tan \alpha\left(\lambda^{2}-1\right)}{\lambda^{2}\left(1+\tan ^{2} \alpha\right) V_{\max }^{2}}, \\
& c=\frac{A^{2}\left(\lambda^{2}+\tan ^{2} \alpha\right)}{\lambda^{2}\left(1+\tan ^{2} \alpha\right) V_{\max }^{2}},
\end{aligned}
$$

it is straightforward to show that the discriminate of the quadratic expression inside $t\left(\xi ; x_{s}\right)$ is always negative

$$
\Delta=b^{2}-4 a c=-\frac{4 A^{2}}{\lambda^{2} V_{\max }^{4}},
$$

and thus the traveltime $t\left(\xi ; x_{s}\right)$ is never null.

Reasoning in the same way as in the isotropic case, the $p$-percentile curve for an elliptical anisotropic medium has the following expressions:

Case 1.

$$
0<x_{s}<\frac{p L}{2}+\frac{b}{2 a} \Longrightarrow m_{p}\left(x_{s}\right)=\sqrt{\frac{\left[2 a\left(x_{s}-p L\right)-b\right]^{2}-\Delta}{4 a}},
$$

Case 2.

$$
\frac{p L}{2}+\frac{b}{2 a}<x_{s}<L-\frac{p L}{2}+\frac{b}{2 a} \Longrightarrow m_{p}\left(x_{s}\right)=\sqrt{\frac{(a p L)^{2}-\Delta}{4 a}},
$$

Case 3.

$$
L-\frac{p L}{2}+\frac{b}{2 a}<x_{s}<L \Longrightarrow m_{p}\left(x_{s}\right)=\sqrt{\frac{\left[2 a\left((1-p) L-x_{s}\right)+b\right]^{2}-\Delta}{4 a}} .
$$

The following can be observed: (i) the percentile curves have a sill whose length is the same than in the isotropic case, but which is shifted a distance equal to $\frac{b}{2 a}=$ $\frac{A\left(\lambda^{2}-1\right) \tan \alpha}{(\lambda \tan \alpha)^{2}+1}$; this effect has already been presented for the mean and the standard deviation curves for anisotropic media (Fernández-Martínez and Pedruelo-González 2008), and (ii) the value of the sill, $m_{p c}=\sqrt{\frac{(a p L)^{2}-\Delta}{4 a}}$, is related to the anisotropic parameters.

\subsection{The Irregular Recording Geometry Case}

The formulas are similar to those developed in the irregular isotropic and rectangular anisotropic cases, but in this case both features act at the same time. A correc- 
tion of the anisotropy angle, $\alpha$, with respect to the $A_{x}$ direction (Fig. 2(a)) is now needed. Deduction is provided in Appendix B. Figure 3(c) shows the comparison of the robust mean traveltime curves for an anisotropic medium with $V_{\max }=5.4 \mathrm{~km} / \mathrm{s}$, $\alpha=45^{\circ}\left(N 45^{\circ} E\right)$, and $\lambda=0.95$, explored through the same irregular recording geometry used in Fig. 2(a). It can be observed that the robust mean traveltime curves shown in this case have similarities to those shown in Figs. 2 and 3(b).

\section{Influence of the Anisotropic Parameters on the Robust Mean Traveltime Curves}

The influence of the anisotropic parameters on the mean curves is explained via synthetic modeling, considering an elliptical anisotropic medium with maximum velocity $5.4 \mathrm{~km} / \mathrm{s}$ and anisotropy direction $N 30^{\circ} \mathrm{W}$. The recording geometry is rectangular, limited by two parallel boreholes of length $L=150$ meters, located 70 meters apart (distance $A$ ) as shown in Fig. 1(a). Figure 4(a) shows the robust mean traveltime curves for anisotropic ratios, $\lambda$, of 0.7 and 0.95 . Anisotropy induces a vertical shift of the percentile curves towards higher values as $\lambda$ decreases; these curves become more asymmetric and may lose one of their branches. Also, horizontal asymptotes may appear in some of the robust MTC curves-inter-quartile range, minimum absolute deviation and standard deviation (Fig. 4(c)). On the other hand, as the parameter $\lambda$ increases, these curves become more symmetric, tending to the isotropic case. Same conclusions are valid for the mad curve, but the vertical shift with respect to the isotropic counterpart is much lower than for the other robust curves. The upper quartile curve is the most sensible to anisotropy type-effects. The other curves are not very different for parameters in the range of weak anisotropy. For the inter-quartile range curve, anisotropy only provokes a lateral shift, i.e., the sill value is constant for any anisotropic ratio. The effect of the anisotropic direction is a lateral shift of the percentile curve, inducing asymmetry. For a source-left recording configuration, the shift is towards the right of the middle point of the line source (70 meters in this case) if the anisotropy direction is northwest, and towards the left if the anisotropy direction is northeast. The shift is the distance $\frac{b}{2 a}$. Finally, increasing $V_{\max }$ lowers the percentile values of the traveltime distribution. These effects are similar to those commented on in Fernández-Martínez and Pedruelo-González (2008) for the mean and the standard deviation curves. Figure 4(b) shows the comparison between the isotropic and anisotropic curves in the range of weak anisotropy $(\lambda=0.95)$. It can be observed that the quartiles are most sensible to anisotropy effects rather than the iqr and mad curves. This result becomes very important for the anisotropic parameter identification.

\subsection{The Effect of Geological Heterogeneities}

The adjustment of the empirical mean traveltime curves, carried over independently on the different mean traveltime curves (robust and classical descriptors) on each region of analysis, is an identification problem whose results might shed light over the causes of data variability. The degree of agreement between the velocity model 


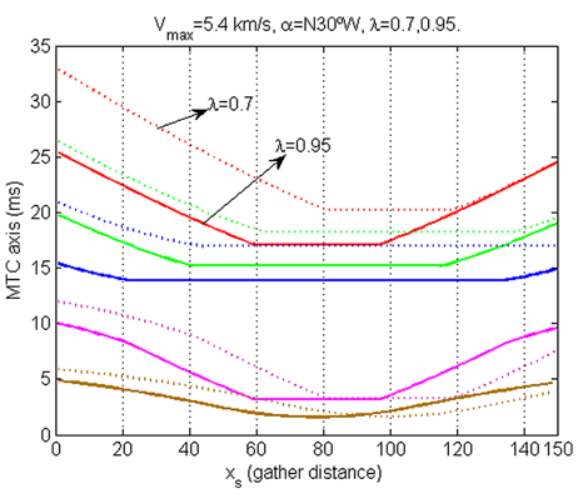

(a)

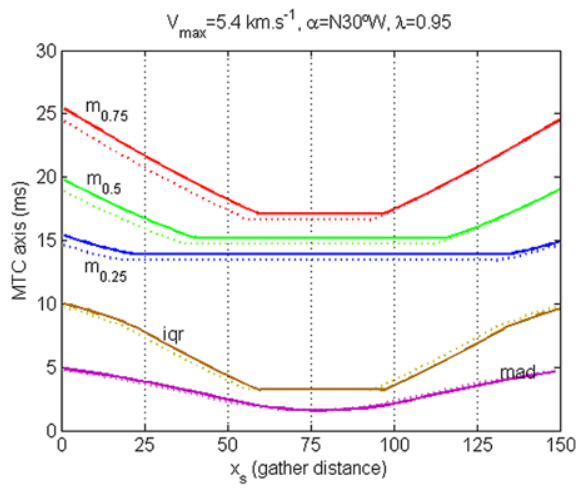

(b)

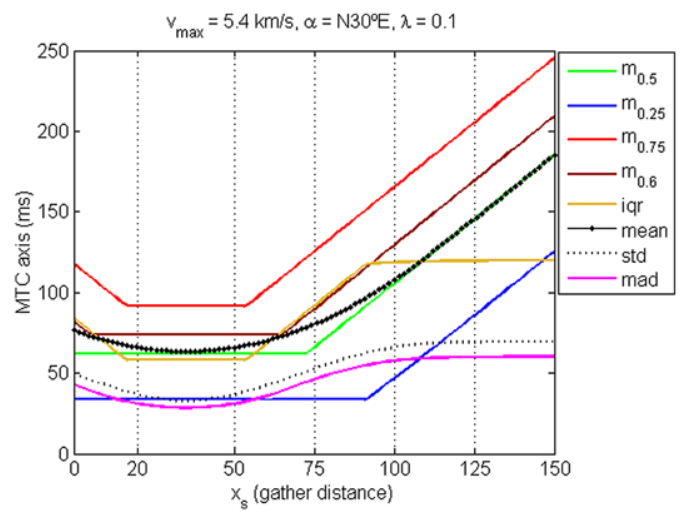

(c)

Fig. 4 Rectangular recording geometry case (source left). (a) Influence of the anisotropic ratio on the robust mean traveltime curves. (b) Comparison to the isotropic curves for an anisotropic medium with $V_{\max }=5.4 \mathrm{~km} / \mathrm{s}, \lambda=0.95$, and $\alpha=30^{\circ}\left(N 60^{\circ} \mathrm{E}\right)$. (c) Robust mean traveltime curves for a medium with $V_{\max }=5.4 \mathrm{~km} / \mathrm{s}, \lambda=0.1$, and $\alpha=30^{\circ}$

estimated from the empirical mean traveltime curves for the shot and the receiver domains provides criteria that help the analysis and the classification of the sources of data variation. This has been investigated for the classical descriptors (mean and standard deviation curves) in Fernández-Martínez et al. (2006). Figure 5 shows the effect of a fault (low velocity anomaly) or an intrusion (high velocity anomaly) zone on the robust mean traveltime curves. The effect of these anomalies is better observed on the lower-quartile and median curves, where a pull-up or pull-down effect can be observed. The std and mad curves in these cases show slope breaks on the boundaries of the velocity heterogeneity. The iqr curves also detect these features, but the effect is much more complicated, inducing two different sills which hamper a clear interpretation of this effect. It can be observed that the effect on the mean and upper-quartile curves is very subtle. 

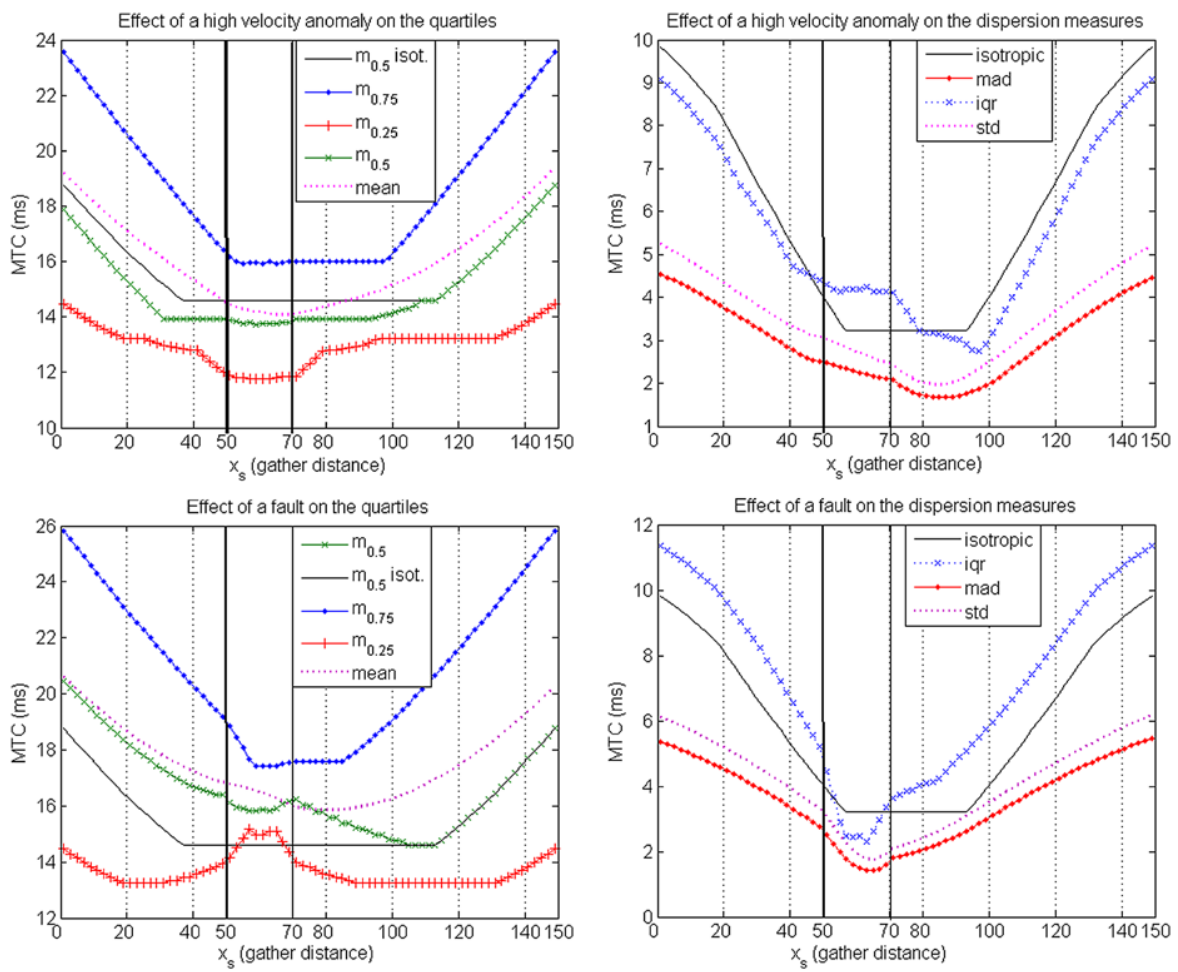

Fig. 5 Effect of an intrusion (high velocity anomaly) and of a fault (low velocity) on the robust mean traveltime curves. Comparison to the isotropic case and to the mean and standard deviation curves

\subsection{Inference of the Anisotropic Velocity Parameters}

The anisotropic parameters $\left(V_{\max }, \alpha, \lambda\right)$ can be inferred from the experimental traveltime counterparts. The method consists in dividing the domain into fairly homogeneous zones (isotropic or anisotropic), and reducing the misfit between the experimental traveltime curves and the theoretical predictions in each gather, solving the following optimization problems of the kind (one for each robust mean time curve in the source and receiver gathers)

$$
\left(V_{\max }, \alpha, \lambda\right)^{*}=\min _{\left(V_{\max }, \alpha, \lambda\right) \in M}\left\|\left(\begin{array}{c}
\bar{t}_{g} \\
\sigma_{g} \\
m_{p} \\
\operatorname{mad} \\
\operatorname{iqr}
\end{array}\right)-\left(\begin{array}{c}
\bar{t}_{g} \\
\sigma_{g} \\
m_{p} \\
\operatorname{mad} \\
\operatorname{iqr}
\end{array}\right)_{\left(V_{\max }, \alpha, \lambda\right)}^{*}\right\|_{2}^{2}
$$

where 


$$
\left(\begin{array}{c}
\bar{t}_{g} \\
\sigma_{g} \\
m_{p} \\
\operatorname{mad} \\
\text { iqr }
\end{array}\right)
$$

are the experimental robust mean traveltime curves in the considered gather (source or receiver), and

$$
\left(\begin{array}{c}
\bar{t}_{g} \\
\sigma_{g} \\
m_{p} \\
\operatorname{mad} \\
\operatorname{iqr}
\end{array}\right)_{\left(V_{\max }, \alpha, \lambda\right)}^{*}
$$

are the corresponding anisotropic robust MTC theoretical predictions for a certain set of anisotropic parameters.

$M$ is the search space for the anisotropic parameters

$$
\begin{aligned}
V_{l} & \leq V_{\max } \leq V_{s}, \\
-90^{\circ} & \leq \alpha \leq 90^{\circ}, \\
0.8 & \leq \lambda \leq 1 .
\end{aligned}
$$

Lower and upper bounds for $V_{\max }$ and the zones of analysis can be deduced from prior information or from the analysis of the isotropic mean traveltime curves themselves, as we have shown for the zonal isotropic case (Fernández-Martínez et al. 2006). Due to the small number of parameters to infer and to the presence of equivalent models and local minima, the optimization is accomplished by means of a global optimization algorithm (Fernández-Martínez et al. 2009a). This methodology is valid for homogeneous anisotropic media at the scale of the experiment (anisotropic single-zone MTC analysis), and also for zonal elliptical anisotropic media (anisotropic multi-zone MTC analysis).

\subsection{Sensitivity Analysis}

To analyze the effect of errors in data in the identification process, we generate a synthetic anisotropic traveltime data set corresponding to a theoretical medium with the following anisotropic parameters $\left(V_{\max }=5.4, \alpha=120^{\circ}, \lambda=0.95\right)$, and times are perturbed by different levels of Gaussian noise. Figure 6 shows the effect of the noise level on the identification of $\left(V_{\max }, \alpha, \lambda\right)$ for the main robust mean traveltime curves, both in the source and receiver gather. The following conclusions may be obtained. First, $V_{\max }$ is the anisotropic parameter that is better identified. This parameter was well identified for noise levels under $10 \%$ in the mean curve and under 5\% in the standard deviation curve (Fernández-Martínez and Pedruelo-González 2008). For high levels of noise, the identification becomes inaccurate originating high velocity artifacts. Second, the anisotropic ratio tends to be underestimated (higher levels 

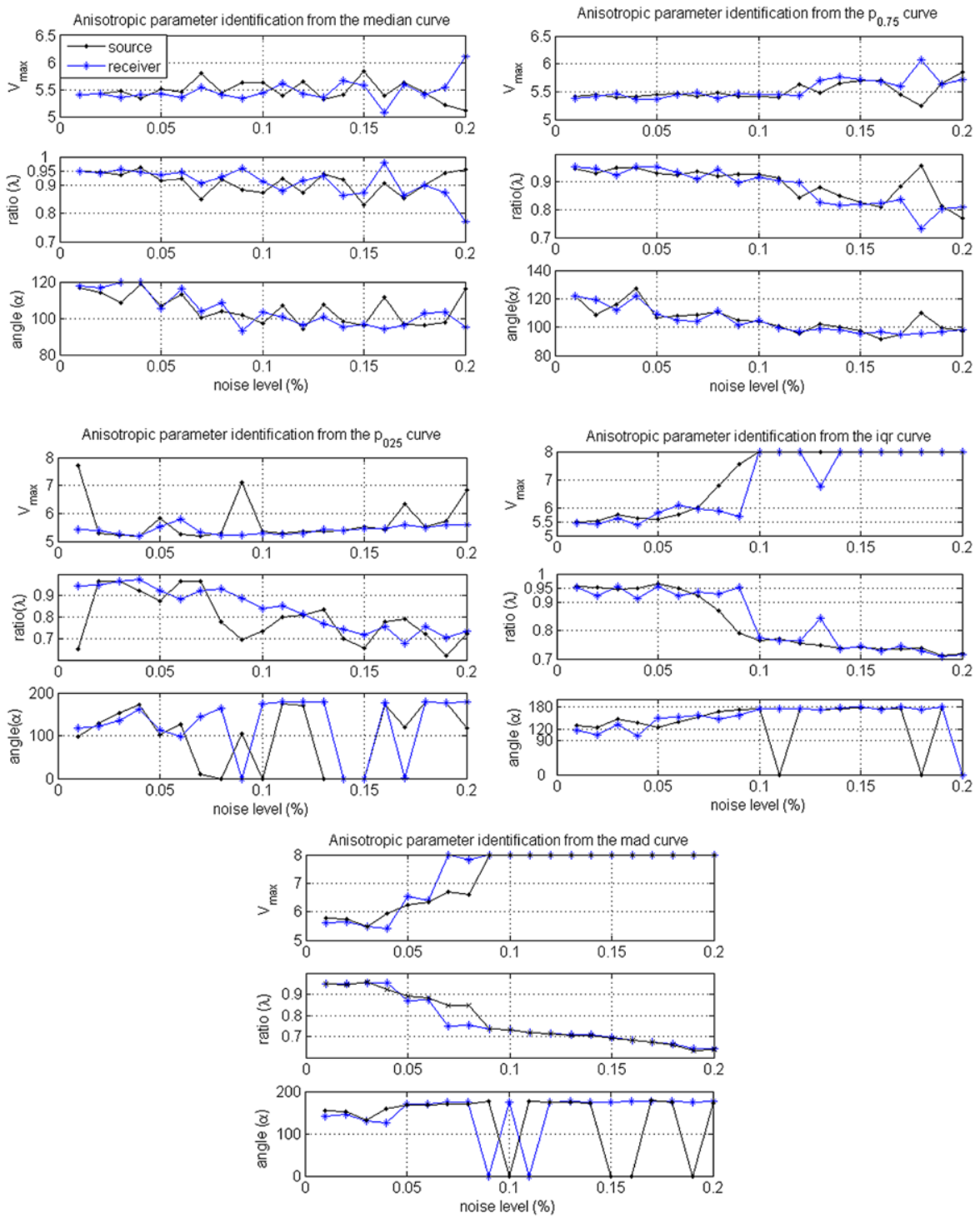

Fig. 6 Effect of the noise on the anisotropic parameter identification for the different robust MTC curves

of anisotropy), and for noise levels higher than $10 \%$ there is a clear degradation of this ratio, which is around 0.7 . Third, generally speaking, the direction of anisotropy is the parameter that is worst identified. For noise levels higher than $8 \%$, this parameter does present a bias towards $180^{\circ}$ (horizontal direction). This behavior is identical in the source and receiver domains. Last, taking into account the above mentioned points, the more robust identification of the anisotropic parameters comes from the median and upper-quartile curves, while the iqr and mad curves are the most sensible to the presence of noise. For instance, the identification of the direction of anisotropy 

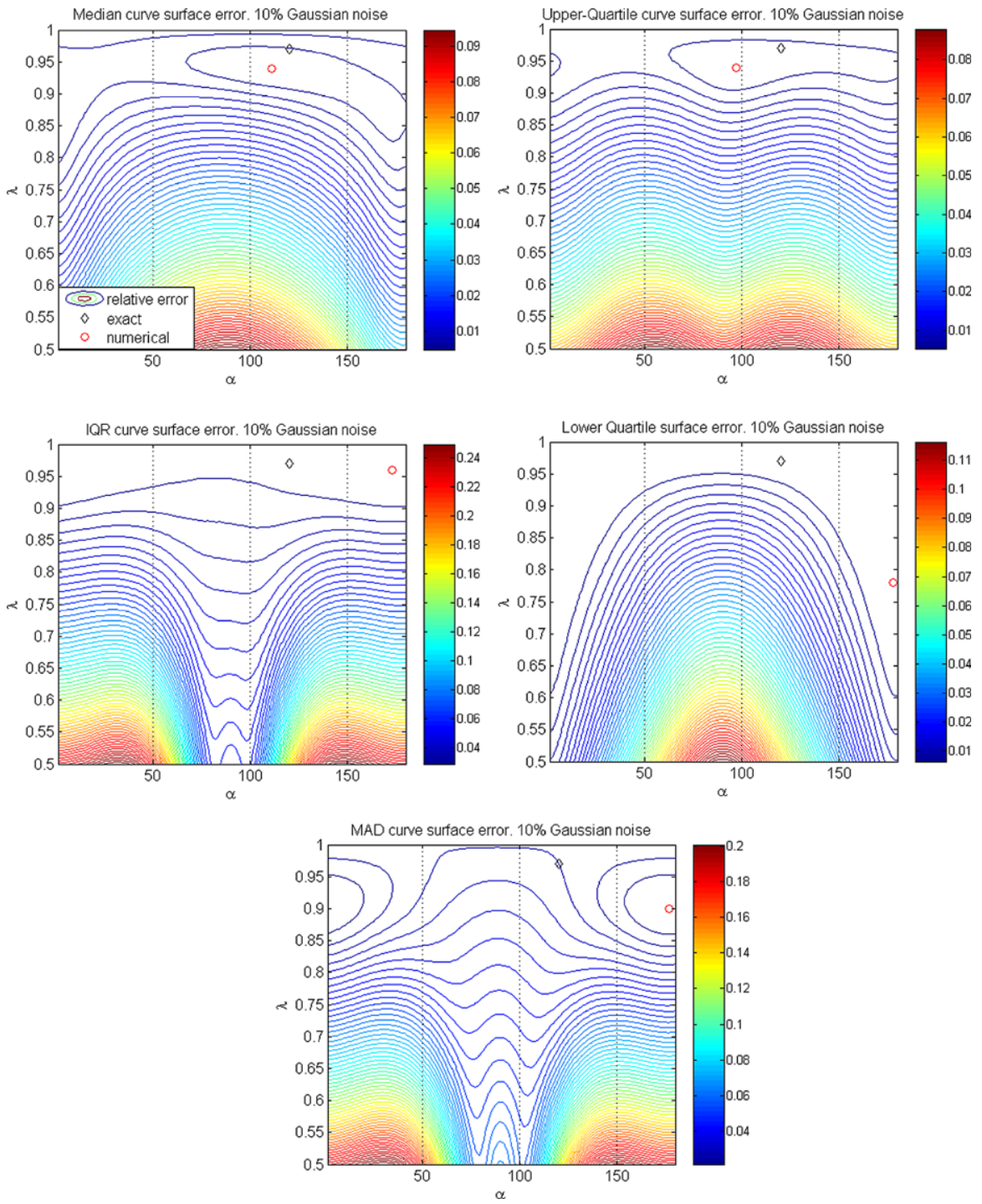

Fig. 7 Effect of the noise on the error surfaces for the different robust MTC curves

from the upper-quartile curve is very robust for very high noise levels. This result is even more important if we take into account the difficulty to identify this parameter from the other curves.

In conclusion, noise makes direction identification ambiguous and biased towards 0 degrees, and generates artifacts in the estimated anisotropy ratios in most of the curves. The median and upper-quartile curves are the most robust descriptors in the presence of noise. These features can be explained analyzing the topography of the misfit surface as a function of $\lambda$ and $\alpha$, assuming that $V_{\max }$ is well constrained. Figure 7 shows the shape of the objective function for the median, upper-quartile, 

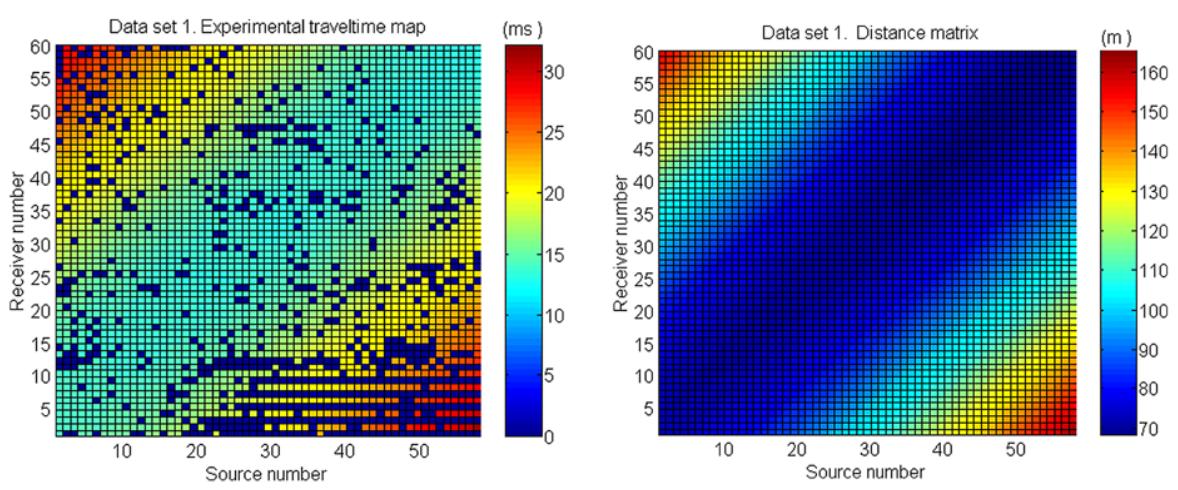

Fig. 8 Experimental traveltime and distance map for the Grimsel data set

lower-quartile and inter-quartile curves for a level of noise of $10 \%$. The region of equivalent models (i.e., the anisotropy sets of parameters which fit equally well the corresponding experimental traveltime curve) is well constrained for the median and upper-quartile curve, whilst for the rest of statistical descriptors (lower quartile, iqr and mad curves) this region becomes broader in size and for some of them presents a central elongated valley parallel to the $\lambda$ axis. This valley deforms progressively as the level of noise increases, becoming a flat elongated area where all the directions give a good fit for a certain range of anisotropic ratios. Thus, when a high level of errors is present, the identification of anisotropic parameters from the lower quartile, inter-quartile and mad curves might become inaccurate.

\section{Study Case: Application to the Grimsel Test Site}

In this section, we show an application of the mean traveltime curves analysis to a data set which comes from an area with granitic geology: the Grimsel test site Field 1 (NAGRA, the Swiss National Cooperative for the disposal of Radioactive Waste).

The geometry of the survey is approximately rectangular, bounded by two boreholes. The dimensions are approximately $70 \times 150 \mathrm{~m}^{2}$. The numbers of sources and receivers are 58 and 60 , and the distance between adjacent sources or receivers is approximately 2.5 meters. The geological and geophysical studies in this granite have been originally undertaken by Gelbke et al. (1989). This data set was also analyzed in Fernández-Martínez et al. (2006, 2008, 2009a) and in Fernández-Martínez and Pedruelo-González (2008), to illustrate the MTC analysis with isotropic and anisotropic assumptions for the velocity model. These authors arrived at the conclusion that this data set was contaminated by a level of approximately $6 \%$ of nonGaussian noise. These authors also inferred the parameters of the acoustic anisotropy, showing via synthetic experiments that errors in data can be misinterpreted as anomalous values of anisotropy (Fernández-Martínez and Pedruelo-González 2008).

The aim in this section is not to perform a complete modeling analysis, but to show how the results issued from the analysis of the robust mean traveltime curves match previous results obtained the classical MTC descriptors (Fernández-Martínez and Pedruelo-González 2008). Figure 8 shows the experimental traveltime and the 

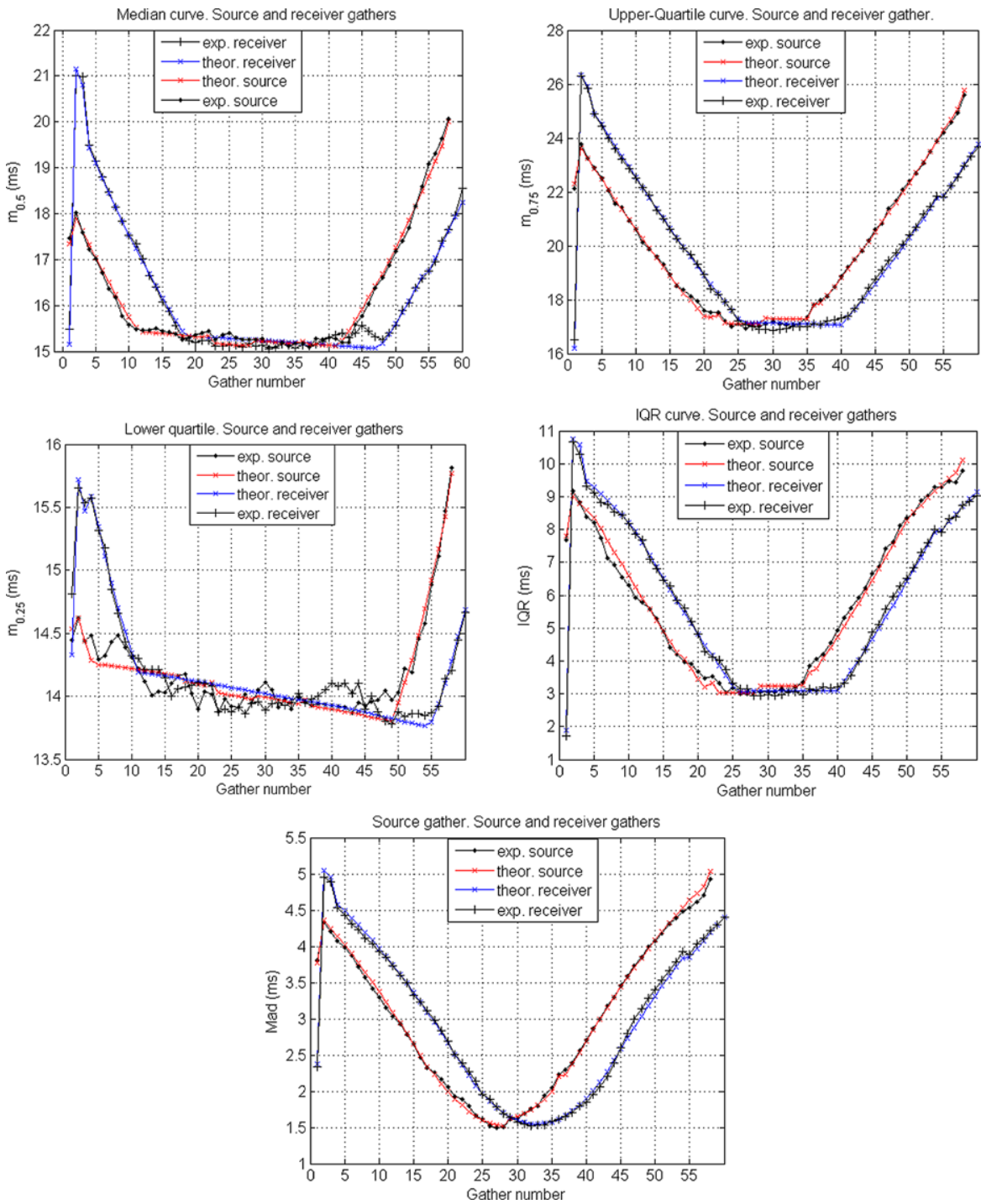

Fig. 9 Experimental and theoretical robust MTC curves for the Grimsel data set (source and receiver gathers). We also show the mean traveltime curve that has been introduced in past publications

acquisition distance maps for this data set. Cells with zero traveltime map indicate pairs of sources and receivers with no available traveltime measurements (mainly receiver gathers 1 to 13) due to acquisition problems. The similarity between both maps indicates behavior close to isotropy and homogeneity. Nevertheless, the experimental traveltime is deformed with respect to the distance map due to the effect of the weak anisotropy. Figure 9 shows the experimental robust mean traveltime curves and their corresponding theoretical models. The corresponding theoretical models are shown in Sect. 3.2. The inference of the anisotropic parameters is developed in Sect. 4.2. It 
Table 1 Grimsel data set 1 . Source Gather. Anisotropic parameters deduced from the robust MTC analysis (degrees measured from the east counter-clockwise)

\begin{tabular}{llllllll}
\hline & Mean & Std & Median & $\begin{array}{l}\text { Upper- } \\
\text { quartile }\end{array}$ & $\begin{array}{l}\text { Lower- } \\
\text { quartile }\end{array}$ & & \\
\hline$V_{\max }(\mathrm{km} / \mathrm{s})$ & $\mathbf{5 . 2 8}$ & 5.64 & $\mathbf{5 . 3 7}$ & $\mathbf{5 . 2 5}$ & 5.21 & 6.37 & 5.76 \\
$\lambda$ & $\mathbf{0 . 9 7}$ & 0.95 & $\mathbf{0 . 9 5}$ & $\mathbf{0 . 9 7}$ & 0.92 & 0.89 & 0.94 \\
$\alpha$ & $\mathbf{6 1}^{\circ}$ & $13^{\circ}$ & $\mathbf{6 5}^{\circ}$ & $\mathbf{7 2}^{\circ}$ & $13^{\circ}$ & $0^{\circ}$ & $8^{\circ}$ \\
\hline
\end{tabular}

Table 2 Grimsel data set 1 . Receiver Gather. Anisotropic parameters deduced from the robust MTC analysis (degrees measured from the east counter-clockwise)

\begin{tabular}{llllllll}
\hline & Mean & Std & Median & $\begin{array}{l}\text { Upper- } \\
\text { quartile }\end{array}$ & $\begin{array}{l}\text { Lower- } \\
\text { quartile }\end{array}$ & & mad \\
\hline$V_{\max }(\mathrm{km} / \mathrm{s})$ & $\mathbf{5 . 2 4}$ & 5.56 & $\mathbf{5 . 3 5}$ & $\mathbf{5 . 2 6}$ & 5.21 & 5.30 & 5.57 \\
$\lambda$ & $\mathbf{0 . 9 7}$ & 0.96 & $\mathbf{0 . 9 5}$ & $\mathbf{0 . 9 7 5}$ & 0.93 & 0.97 & 0.96 \\
$\alpha$ & $\mathbf{2 8}^{\circ}$ & $25^{\circ}$ & $\mathbf{6 5}^{\circ}$ & $\mathbf{5 1}^{\circ}$ & $11^{\circ}$ & $56^{\circ}$ & $25^{\circ}$ \\
\hline
\end{tabular}

can be observed that there is a good match between them in all the cases. The lower quartile curve is the one that presents more low scale variabilities, which can be due partially to the effect of noise.

Tables 1 and 2 show the results of this analysis for the source and receiver gathers. These tables show the maximum velocity, direction and ratio of anisotropy deduced from the analysis and fitting of the different mean time descriptors (Sect. 4.2). We outline in bold the parameters inferred from the mean, median, and upper-quartile curves. Results are very coherent for these descriptors in the source and receiver gathers, since they are less prone to errors than the standard deviation, inter-quartile range, lower quartile and minimum absolute deviation. This situation agrees with the conclusions that have been presented on the section devoted to the sensibility analysis of the robust mean traveltime descriptors via synthetic experiments.

\section{Conclusions}

In the present work, we have presented the mathematical deduction and analysis of robust traveltime curves in elliptical anisotropic media, which complete the analysis presented in the past for the mean and standard deviation curves. The fitting of the empirical traveltime curves provide us with a new a simple method to estimate the model of elliptical anisotropy from traveltime data before the inversion, and to analyze the traveltime quality. The mean, median, and upper-quartile curves are the most robust descriptors to identify anisotropic parameters. Conversely, the rest of descriptors are very sensible to data errors, and they can be used to detect their presence before the inversion. This filtering process is very important because we have shown that data errors provoke anisotropy artifacts, very anomalous anisotropic ratios and wrong directions of anisotropy biased towards 0 degrees. This is a very important conclusion, since it highlights that data errors can be interpreted as false anisotropy. Thus, performing mean traveltime curve analysis is always recommended, since it does not need stringent numerical requirements. 
Acknowledgements We would like to thank NAGRA for the permission to use the Grimsel data set to illustrate this methodology. This work benefited from a sabbatical grant at the University of California Berkeley (Department of Civil and Environmental Engineering) given by the University of Oviedo (Spain), and by the "Secretaría de Estado de Universidades y de Investigación" of the Spanish Ministry of Science and Innovation. We also acknowledge the financial support for the present academic year coming from the University of California Berkeley and the Lawrence Berkeley National Laboratory (Earth Science Division), and from the Energy Resources Engineering Department of Stanford University (Stanford Center for Reservoir Forecasting and Smart Field Consortiums).

Open Access This article is distributed under the terms of the Creative Commons Attribution Noncommercial License which permits any noncommercial use, distribution, and reproduction in any medium, provided the original author(s) and source are credited.

\section{Appendix A: Isotropic Medium Explored via an Irregular Recording Geometry}

These formulas correspond to Sect. 2.2. The variables that appear in the following formulas are those presented in Fig. 2(a).

\section{A.1 $p$-Percentiles and the mad Curves}

Case 1.

$$
\begin{aligned}
0<s_{r}<\frac{p L_{r}}{2}, \quad \text { that is, } \\
0<x_{s}<\frac{(p-1) L_{r}^{2}-d_{0}^{2}+L_{s}^{2}+d_{n}^{2}-2 L_{s} d_{n} \cos \gamma}{2\left(L_{s}-d_{n} \cos \gamma-d_{0} \cos \delta\right)}, \\
m_{p}\left(x_{s}\right)=\sqrt{\frac{\left(p L_{r}-s_{r}\right)^{2}+A_{x}^{2}}{V^{2}}}, \\
\operatorname{mad}\left(x_{s}\right)=m_{p}(2 p-1)+\bar{t}_{\left[0, L_{r}\right]}-2 p \bar{t}_{\left[0, L_{r} p\right]},
\end{aligned}
$$

Case 2.

$$
\begin{aligned}
& \frac{p L_{r}}{2}<s_{r}<L_{r}-\frac{p L_{r}}{2}, \quad \text { that is, } \\
& \frac{(p-1) L_{r}^{2}-d_{0}^{2}+L_{s}^{2}+d_{n}^{2}-2 L_{s} d_{n} \cos \gamma}{2\left(L_{s}-d_{n} \cos \gamma-d_{0} \cos \delta\right)} \\
& <x_{s}<\frac{(1-p) L_{r}^{2}-d_{0}^{2}+L_{s}^{2}+d_{n}^{2}-2 L_{s} d_{n} \cos \gamma}{2\left(L_{s}-d_{n} \cos \gamma-d_{0} \cos \delta\right)}, \\
& m_{p}\left(x_{s}\right)=\sqrt{\frac{\left(\frac{p L_{r}}{2}\right)^{2}+A_{x}^{2}}{V^{2}}}, \\
& \operatorname{mad}\left(x_{s}\right)=m_{p}(2 p-1)+\bar{t}_{\left[0, L_{r}\right]}-2 p \bar{t}_{\left[s_{r}-\frac{L_{r} p}{2}, s_{r}+\frac{L_{r} p}{2}\right]},
\end{aligned}
$$


Case 3.

$$
\begin{aligned}
& L_{r}-\frac{p L_{r}}{2}<s_{r}<L_{r}, \quad \text { that is, } \\
& \frac{(1-p) L_{r}^{2}-d_{0}^{2}+L_{s}^{2}+d_{n}^{2}-2 L_{s} d_{n} \cos \gamma}{2\left(L_{s}-d_{n} \cos \gamma-d_{0} \cos \delta\right)}<x_{s}<L_{s}, \\
& m_{p}\left(x_{s}\right)=\sqrt{\frac{\left((p-1) L_{r}+s_{r}\right)^{2}+A_{x}^{2}}{V^{2}}}, \\
& \operatorname{mad}\left(x_{s}\right)=m_{p}(2 p-1)+\bar{t}_{\left[0, L_{r}\right]}-2 p \bar{t}_{\left[L_{r}(1-p), L_{r}\right]} .
\end{aligned}
$$

\section{A.2 Inter-Quartile Range}

Case 1.

$$
\begin{aligned}
0<s_{r}<\frac{L_{r}}{8}, \quad \text { that is, } \\
0<x_{s}<\frac{-3 L_{r}^{2}+4\left(L_{s}^{2}-d_{0}^{2}+d_{n}^{2}-2 L_{s} d_{n} \cos \gamma\right)}{8\left(L_{s}-d_{n} \cos \gamma-d_{0} \cos \delta\right)} \\
\Longrightarrow \operatorname{iqr}\left(x_{s}\right)=\sqrt{\frac{\left(\frac{3 L_{r}}{4}-s_{r}\right)^{2}+A_{x}^{2}}{V^{2}}}-\sqrt{\frac{\left(\frac{L_{r}}{4}-s_{r}\right)^{2}+A_{x}^{2}}{V^{2}}},
\end{aligned}
$$

Case 2.

$$
\begin{aligned}
& \frac{L}{8}<s_{r}<\frac{3 L_{r}}{8}, \quad \text { that is, } \\
& \frac{-3 L_{r}^{2}+4\left(L_{s}^{2}-d_{0}^{2}+d_{n}^{2}-2 L_{s} d_{n} \cos \gamma\right)}{8\left(L_{s}-d_{n} \cos \gamma-d_{0} \cos \delta\right)} \\
& \quad<x_{s}<\frac{-L_{r}^{2}+4\left(L_{s}^{2}-d_{0}^{2}+d_{n}^{2}-2 L_{s} d_{n} \cos \gamma\right)}{8\left(L_{s}-d_{n} \cos \gamma-d_{0} \cos \delta\right)}, \\
& \operatorname{iqr}\left(x_{s}\right)=\sqrt{\frac{\left(\frac{3 L_{r}}{4}-s_{r}\right)^{2}+A_{x}^{2}}{V^{2}}}-\sqrt{\frac{\left(\frac{L_{r}}{8}\right)^{2}+A_{x}^{2}}{V^{2}}},
\end{aligned}
$$

Case 3.

$$
\begin{aligned}
& \frac{3 L_{r}}{8}<s_{r}<\frac{5 L_{r}}{8}, \text { that is, } \\
& \frac{-L_{r}^{2}+4\left(L_{s}^{2}-d_{0}^{2}+d_{n}^{2}-2 L_{s} d_{n} \cos \gamma\right)}{8\left(L_{s}-d_{n} \cos \gamma-d_{0} \cos \delta\right)} \\
& <x_{s}<\frac{L_{r}^{2}+4\left(L_{s}^{2}-d_{0}^{2}+d_{n}^{2}-2 L_{s} d_{n} \cos \gamma\right)}{8\left(L_{s}-d_{n} \cos \gamma-d_{0} \cos \delta\right)}, \\
& \operatorname{iqr}\left(x_{s}\right)=\sqrt{\frac{\left(\frac{3 L_{r}}{8}\right)^{2}+A_{x}^{2}}{V^{2}}}-\sqrt{\frac{\left(\frac{L_{r}}{8}\right)^{2}+A_{x}^{2}}{V^{2}}},
\end{aligned}
$$


Case 4.

$$
\begin{aligned}
& \frac{5 L_{r}}{8}<s_{r}<\frac{7 L_{r}}{8}, \quad \text { that is, } \\
& \frac{L_{r}^{2}+4\left(L_{s}^{2}-d_{0}^{2}+d_{n}^{2}-2 L_{s} d_{n} \cos \gamma\right)}{8\left(L_{s}-d_{n} \cos \gamma-d_{0} \cos \delta\right)} \\
& \quad<x_{s}<\frac{3 L_{r}^{2}+4\left(L_{s}^{2}-d_{0}^{2}+d_{n}^{2}-2 L_{s} d_{n} \cos \gamma\right)}{8\left(L_{s}-d_{n} \cos \gamma-d_{0} \cos \delta\right)}, \\
& \operatorname{iqr}\left(x_{s}\right)=\sqrt{\frac{\left(s_{r}-\frac{L_{r}}{4}\right)^{2}+A_{x}^{2}}{V^{2}}}-\sqrt{\frac{\left(\frac{L_{r}}{8}\right)^{2}+A_{x}^{2}}{V^{2}}},
\end{aligned}
$$

Case 5.

$$
\begin{aligned}
& \frac{7 L_{r}}{8}<s_{r}<L_{r}, \quad \text { that is, } \\
& \frac{3 L_{r}^{2}+4\left(L_{s}^{2}-d_{0}^{2}+d_{n}^{2}-2 L_{s} d_{n} \cos \gamma\right)}{8\left(L_{s}-d_{n} \cos \gamma-d_{0} \cos \delta\right)}<x_{s}<L_{s}, \\
& \operatorname{iqr}\left(x_{s}\right)=\sqrt{\frac{\left(s_{r}-\frac{L_{r}}{4}\right)^{2}+A_{x}^{2}}{V^{2}}}-\sqrt{\frac{\left(s_{r}-\frac{3 L_{r}}{4}\right)^{2}+A_{x}^{2}}{V^{2}}} .
\end{aligned}
$$

\section{Appendix B: Elliptical Anisotropic Medium Explored via an Irregular Recording Geometry}

These formulas correspond to Sect. 3.2. In this case, for the sake of simplicity, we omit the formulas describing the inter-quartile range, since they do not provide additional insights.

B.1 $p$-Percentiles and the mad Curve

Case 1.

$$
\begin{aligned}
0<s_{r}<\frac{p L_{r}}{2}+\frac{b}{2 a}, \quad \text { that is, } \\
0<x_{s}<\frac{(p-1) L_{r}^{2} a+L_{r} b-a\left(d_{0}^{2}-L_{s}^{2}-d_{n}^{2}+2 L_{s} d_{n} \cos \gamma\right)}{2 a\left(L_{s}-d_{n} \cos \gamma-d_{0} \cos \delta\right)}, \\
m_{p}\left(x_{s}\right)=\sqrt{\frac{\left[\left(p L_{r}-s_{r}\right) 2 a+b\right]^{2}-\Delta}{4 a}}, \\
\operatorname{mad}\left(x_{s}\right)=m_{p}(2 p-1)+\bar{t}_{\left[0, L_{r}\right]}-2 p \bar{t}_{\left[0, L_{r} p\right]},
\end{aligned}
$$


Case 2.

$$
\begin{aligned}
& \frac{p L_{r}}{2}+\frac{b}{2 a}<s_{r}<L_{r}-\frac{p L_{r}}{2}+\frac{b}{2 a}, \quad \text { that is, } \\
& x_{s}>\frac{(p-1) L_{r}^{2} a+L_{r} b-a\left(d_{0}^{2}-L_{s}^{2}-d_{n}^{2}+2 L_{s} d_{n} \cos \gamma\right)}{2 a\left(L_{s}-d_{n} \cos \gamma-d_{0} \cos \delta\right)} \text { and } \\
& x_{s}<\frac{(p-1) L_{r}^{2} a-L_{r} b+a\left(d_{0}^{2}-L_{s}^{2}-d_{n}^{2}+2 L_{s} d_{n} \cos \gamma\right)}{2 a\left(d_{n} \cos \gamma+d_{0} \cos \delta-L_{s}\right)}, \\
& m_{p}\left(x_{s}\right)=\sqrt{\frac{\left(a p L_{r}\right)^{2}-\Delta}{4 a}}, \\
& \operatorname{mad}\left(x_{s}\right)=m_{p}(2 p-1)+\bar{t}_{\left[0, L_{r}\right]}-2 p \bar{t}_{\left[s_{r}-\frac{L_{r} p}{2}-\frac{b}{2 a}, s_{r}+\frac{L_{r} p}{2}-\frac{b}{2 a}\right]},
\end{aligned}
$$

Case 3.

$$
\begin{aligned}
& L_{r}-\frac{p L_{r}}{2}+\frac{b}{2 a}<s_{r}<L_{r}, \quad \text { that is, } \\
& \frac{(p-1) L_{r}^{2} a-L_{r} b+a\left(d_{0}^{2}-L_{s}^{2}-d_{n}^{2}+2 L_{s} d_{n} \cos \gamma\right)}{2 a\left(d_{n} \cos \gamma+d_{0} \cos \delta-L_{s}\right)}<x_{s}<L_{s}, \\
& m_{p}\left(x_{s}\right)=\sqrt{\frac{\left[2 a\left((1-p) L-s_{r}\right)+b\right]^{2}-\Delta}{4 a}}, \\
& \operatorname{mad}\left(x_{s}\right)=m_{p}(2 p-1)+\bar{t}_{\left[0, L_{r}\right]}-2 p \bar{t}_{\left[L_{r}(1-p), L_{r}\right] .}
\end{aligned}
$$

\section{References}

Berryman JG (1991) Lectures notes on nonlinear inversion and tomography. Borehole seismic tomography. Technical report, Lawrence Livermore National Laboratory

Dines KA, Lytle RJ (1979) Computerized geophysical tomography. IEEE Proc 67(7):1065-1073

Fernández-Martínez JL, Pedruelo-González LM (2008) Anisotropic mean traveltime curves: a method to estimate anisotropic parameters from 2D transmission tomographic data. Math Geosci 41(2):163192. doi:10.1007/s11004-008-9202-4

Fernández-Martínez JL, Fernández-Álvarez JP, Pedruelo-González LM (2006) Mean traveltime curves analysis: a method to improve understanding of data behaviour in 2-D transmission tomography at the pre-inversion stage. Math Geol 38(3):343-374

Fernández-Martínez JL, Fernández-Alvarez JP, Pedruelo-González LM (2008) MTCLAB: a MATLAB ${ }^{\circledR}$ based program for traveltime quality analysis and preinversion velocity tuning in 2-D transmission tomography. Comput Geosci 34(3):213-225. doi:10.1016/j.cageo.2007.03.008

Fernández-Martínez JL, Pedruelo-González LM, García-Gonzalo E (2009a) AMTCLAB: a MATLAB ${ }^{\circledR}$ based program for traveltime analysis and velocity tuning in $2 \mathrm{D}$ elliptical anisotropic media. Comput Geosci 35(10):2057-2064. doi:10.1016/j.cageo.2008.11.013

Fernández-Martínez JL, Pedruelo-González LM, DeParis J, Mangriotis MD, Rector JW (2009b). Application of the mean traveltime curves to GPR and VSP data. In: Annual meeting, EEGS, SAGEEP

Gelbke C, Miranda F, Sattel G (1989) The results of a seismic transmission tomography survey at the Grimsel rock laboratory. Log Anal 243-260 
Gordon R (1974) A tutorial on ART. IEEE Trans Nucl Sci NS-21:78-93

Ivansson S (1986) Seismic borehole tomography. Theory and computational methods. IEEE Proc 74(2):328-338

Scales JA (1987) Tomographic inversion via the conjugate gradient method. Geophysics 52:179-185

Scales JA, Gersztenkorn A (1988) Robust methods in inverse theory. Inverse Probl 4:1071-1091 